\title{
THE FIELD THEORETICAL APPROACH TO STUDY OF THE CRITICAL BEHAVIOUR OF THE WEAKLY DILUTED QUENCHED ISING MODEL IN GENERAL DIMENSIONS. THREE-LOOP APPROXIMATION
}

\author{
YU.HoLOVATCH ${ }^{(1)}$, T.YAVORS'KII ${ }^{(2)}$ \\ (1) Institute for Condensed Matter Physics \\ of the Ukrainian Academy of Sciences \\ 1 Svientsitskii St., UA-290011 Lviv-11, Ukraine \\ ${ }^{(2)}$ Department of Theoretical Physics, \\ Ivan Franko Lviv State Univesity \\ 12 Drahomanov str., UA-290005 Lviv-11, Ukraine
}

Received November 6, 1997

\begin{abstract}
Within the fixed-dimension field theoretical renormalization group approach the expressions for the $\beta$ - and $\gamma$-functions of the anisotropic $m n$-vector model are obtained in the three-loop approximation. Critical exponents for the most interesting case of the weakly diluted quenched Ising model, as well as estimates for the marginal order parameter component number $m_{c}$ are calculated as functions of $d$ in the region $2 \leqslant d<4$. Conclusions concerning the effectiveness of different resummation techniques are drawn. The results are compared with avaliable data of recent studies.
\end{abstract}

\section{Introduction}

There are at least two quite different possibilities to approach the concept of non-integer dimensionality. The first one is to consider a function which characterizes numerically an object on a line or in the space to be defined not only for natural, but also for any real value of space dimension. This implies that one is carrying out an analytic continuation of the function, and besides, neither the space of non-integer dimension nor the object in it is specified. The second possibility, less formal, consists in the explicit definition of the non-integer dimensional object itself. This way of extending beyond Euclidean geometry within the sphere of physical interest was realized by introducing the concept of a fractal [1].

Both of the stated above ideas were reflected in the theory of critical phenomena. Thus, the first one was embodied in studying many-particle systems on abstract hypercubic lattices of the non-integer dimension $(d)$, e.g. by constructing $\epsilon$-expansion [2], the second one - in examining their critical behaviour on fractal lattices [3,4]. Since two ways of generalizing appeared, there arose a question if a model on a fractal lattice (being scale invariant) possesses universality, as well as a system on a hypercubic lattice (having translation invariance). The question has been widely studied but still remains open [5-8]. Today's point of view states that the usual demand for "strong universality" (in sense of critical properties depending only on

(C) Yu.Holovatch, T.Yavors'kii, 1997

ISSN 0452-9910. Condensed Matter Physics 1997 No 11 (87-108) 
symmetry of the order parameter, interaction range and space dimension) seems not to be obeyed by fractal lattice systems, and for them the concept of universality itself should be revised $[9,10]$. However, some kinds of spin systems on fractal lattices may interpolate hypercubic lattices results [11].

Now the notion of the non-integer space dimension $d$ is in common use in the theory of critical phenomena. Speaking about the studies of Ising-like models in non-integer $d$, one should note a great variety of theoretical approaches devised for these problems. These include: the mentioned WilsonFisher $\epsilon$-expansion [2] improved by the summation method [12]; Kadanoff lower-bound renormalization applied to some special non-integer dimensions [13]; high-temperature expansion improved by a variation technique [14]; finite-size scaling method applied to numerical transfer-matrices data $[15,16]$; new perturbation theory based on the physical branch of the solution of the renormalization group equation [17-20]; fixed dimension renormalization group technique $[21,22]$ applied directly to arbitrary non-integer $d[23,24]$.

Perhaps the first paper devoted to the study of the Ising model in different, however not non-integer dimension, was [25] where non-universal properties of the model were discussed.

All these approaches, as well as computer simulations, confirm the correctness of the universality hypothesis also for non-integer $d$ hypercubic lattices and allow us to obtain the critical exponents as functions of $d$ with high accuracy.

Returning to the study of the critical behaviour at integer $d$, one should note that the problem becomes more complicated when studying spin systems with a structural disorder. Whereas the case of the annealed disorder is of less interest from the point of view of determining asymptotical values of critical exponents [26], the weak quenched disorder has been a subject of intensive study. Here the Harris criterion [28] has been devised. It states that if the heat capacity exponent $\alpha_{\text {pure }}$ of a pure model is negative, that is the heat capacity has no divergence at the critical point, impurities do not affect the critical behaviour of the model in the sense that critical exponents remain unchanged under dilution. But if only $\alpha_{\text {pure }}>0$, then the critical behaviour of the disordered model is governed by a new set of critical exponents. As far as for a $3 d m$-vector spin model only the $3 d$ Ising model $(m=1)$ is characterized by $\alpha_{\text {pure }}>0$, it is the Ising model which is of special interest. And because of the triviality of the annealed disorder in the sense mentioned above, the most interesting object for study is just the quenched Ising model. The appearance of a set of new critical exponents for that model at $d=3$ is confirmed by the experiments [29-31], renormalization group $(R G)$ calculations [32-40,23,41], Monte-Carlo $(M C)$ [42-46] and $R G M C$ [47] simulations.

The situation is not so simple for the $2 d$ Ising model. Onsager exact solution of the pure model proves the logarithmic divergence of heat capacity, which yields $\alpha_{\text {pure }}=0$, and allows one, in accordance with the Harris criterion, to clasify this case as a marginal one. Most of the theoretical works suggest that the $2 d$ Ising model with a quenched disorder has the same critical behaviour as the $2 d$ pure Ising model (except for logarithmic corrections) [48-52,39,40,53] (see also review [54]). This result is corroborated by $M C$ simulations on two-dimensional lattices [55-59] and experiments $[60,61]$.

Deviations from the expected critical exponents, which sometimes are observed during such computations, are explained by a system being not in the asymptotic region (see [59] for recent study). Nevertheless, some authors assert that for the $2 d$ Ising model with a quenched disorder a new 
critical behaviour appears $[62,63]$

While the undiluted Ising model at non-integer $d$ was a subject of intensive study [2,12-20], it is not the case for the diluted Ising model. We can only mention here the work [36], where the model was studied within the Golner-Riedel scaling field [64] approach. It is worthwhile to note that the $\varepsilon$-expansion technique applied to this model, due to the fact that $R G$ equations appear to be degenerated on the one loop level, results in $\sqrt{\varepsilon}$ expansion for the critical exponents [34]. The latter is known up to the $\varepsilon^{3 / 2}$ order $[65,66]$. Most likely this expansion is not asymptotic and thus does not yield reliable quantitative data for $d$ far enough from $d=4$ [67]. The equations of the massive field theory at fixed integer $d[21,22]$ first applied to the diluted Ising model at $d=2,3$ in $[35,37]$ were found to be the most effective method for investigating this problem. In order to consider an arbitrary non-integer $d$ the Parisi approach [21,22] was generalized in [68] where critical behaviour of the model was studied in a two-loop approximation. The aim of the present work, based on the massive field theoretical approach, is to make a more detailed investigation of the critical behaviour of the diluted $O(m)$-vector model at arbitrary $d$. Though it is the case $m=1$ in which we are interested most of all, we consider the $R G$-equations for any $m$, which also allow us to study the crossover in the model at any $d$. We will obtain the $R G$-equations within the 3-loop approximation and apply to their analysis different resummation procedures in order to find the most reliable one.

The set-up of the article is as follows. In the next Section we introduce the model and the notation. Then we describe the $R G$-procedure adopted here and give the series for the $R G$-functions of the weakly diluted quenched $m$-vector model in the three-loop approximation. Being asymptotic, these series are to be resummed. This is done in Section 2 where different ways of resummation are used. Section 3 concludes our study giving results for the quantitative characteristics of the critical behaviour and discussing them. In the Conclusions we give some general comments to the present work. In the Appendix we list some lengthy expressions for the coefficients of the $R G$-functions in the three-loop approximation.

\section{The Model and the $R G$-procedure}

As it is well known, the critical behaviour of the quenched weakly-diluted $m$ - vector model is governed by a Lagrangian with two coupling constants [34]:

$$
\begin{aligned}
\mathcal{L}(\phi)= & \int \mathrm{d}^{d} R\left\{\frac{1}{2} \sum_{\alpha=1}^{n}\left[\left|\nabla \vec{\phi}^{\alpha}\right|^{2}+m_{0}^{2}\left|\vec{\phi}^{\alpha}\right|^{2}\right]+\frac{u_{0}}{4 !}\left(\sum_{\alpha=1}^{n}\left|\vec{\phi}^{\alpha}\right|^{2}\right)^{2}+\right. \\
& \left.\frac{v_{0}}{4 !} \sum_{\alpha=1}^{n}\left(\left|\vec{\phi}^{\alpha}\right|^{2}\right)^{2}\right\}
\end{aligned}
$$

in replica limit $n \rightarrow 0$. Here any $\vec{\phi}^{\alpha}$ is a $m$-component vector

$\vec{\phi}^{\alpha}=\left(\phi^{\alpha, 1}, \phi^{\alpha, 2}, \ldots, \phi^{\alpha, m}\right) ; u_{0}>0, v_{0}<0$ are bare coupling constants; $m_{0}$ is bare mass.

As it was already stated above, we adopt here the renormalized massive field theory scheme $[21,22]$ in order to extract the critical behaviour governed by (2.1). We start from the defined by (2.1) unrenormalized one-particle 
irreducible vertex functions

$$
\Gamma^{(L, N)}\left(q_{1}, . ., q_{L} ; p_{1}, . ., p_{N} ; m_{0}, u_{0}, v_{0} ; \Lambda_{0} ; d\right)
$$

depending on the wave vectors $\{q\},\{p\}$, bare parameters $m_{0}, u_{0}, v_{0}$ and the ultraviolet momentum cutoff $\Lambda_{0}$. The vertex functions' dependence on the space dimension $d$ is explicitly noted here as well. We impose the renormalization conditions at zero external momenta and non-zero mass (see $[69,70]$ for instance) at the limit $\Lambda_{0} \rightarrow \infty$ for the renormalized functions [71] $\Gamma_{R}^{(0,2)}, \Gamma_{R, u}^{(0,4)}, \Gamma_{R, v}^{(0,4)}, \Gamma_{R}^{(1,2)}$ :

$$
\begin{aligned}
\left.\Gamma_{R}^{(0,2)}(p,-p ; m, u, v ; d)\right|_{p=0} & =m^{2}, \\
\left.\frac{d}{d p^{2}} \Gamma_{R}^{(0,2)}(p,-p ; m, u, v ; d)\right|_{p=0} & =1 \\
\left.\Gamma_{R, u}^{(0,4)}\left(\left\{p_{i}\right\} ; m, u, v ; d\right)\right|_{\left\{p_{i}\right\}=0} & =m^{4-d} u \\
\left.\Gamma_{R, v}^{(0,4)}\left(\left\{p_{i}\right\} ; m, u, v ; d\right)\right|_{\left\{p_{i}\right\}=0} & =m^{4-d} v \\
\left.\Gamma_{R, u}^{(1,2)}(q ; p,-p ; m, u, v ; d)\right|_{q=p=0} & =1,
\end{aligned}
$$

with $m, u, v$ being the renormalized mass $m=Z_{3} m_{1}=Z_{3} \Gamma^{(0,2)}\left(0 ; m_{0}, u_{0}, v_{0}\right)$ and couplings $u=m^{d-4} Z_{3}^{2} Z_{1, u}^{-1} u_{0}, v=m^{d-4} Z_{3}^{2} Z_{1, v}^{-1} v_{0}$. From these conditions there follow expansions for the renormalized constants for field $\left(Z_{3}\right)$, vertices $u\left(Z_{1, u}\right), v\left(Z_{1, v}\right)$ and $\phi^{2}$ insertion $\left(Z_{2}\right)$. Subsequently, these define the coefficients $\beta, \gamma$ entering the corresponding Callan-Symanzik equation:

$$
\begin{aligned}
\beta_{u}(u, v) & =\left.\frac{\partial u}{\partial \ln m}\right|_{u_{0}, v_{0}}, \\
\beta_{v}(u, v) & =\left.\frac{\partial v}{\partial \ln m}\right|_{u_{0}, v_{0}}, \\
\gamma_{\phi} \equiv \gamma_{3} & =\left.\frac{\partial Z_{3}}{\partial \ln m}\right|_{u_{0}, v_{0}}, \\
\bar{\gamma}_{\phi^{2}} \equiv \gamma_{2} & =-\left.\frac{\partial Z_{2}}{\partial \ln m}\right|_{u_{0}, v_{0}} .
\end{aligned}
$$

In the stable fixed point $\left\{u^{*}, v^{*}\right\}$ to be defined by a simultaneous zero of the both $\beta$-functions:

$$
\begin{aligned}
& \beta_{u}\left(u^{*}, v^{*}\right)=0 \\
& \beta_{v}\left(u^{*}, v^{*}\right)=0
\end{aligned}
$$

the $\gamma_{\phi}$-function gives the critical exponent $\eta$ of the pair correlation function:

$$
\gamma_{\phi}\left(u^{*}, v^{*}\right)=\eta .
$$

The correlation length critical exponent $\nu$ is defined in the stable fixed point by:

$$
\bar{\gamma}_{\phi^{2}}\left(u^{*}, v^{*}\right)=2-\nu^{-1}-\gamma_{\phi}\left(u^{*}, v^{*}\right) .
$$

Using familiar scaling relations, one can easily calculate any other critical exponents on the basis of $\eta$ and $\nu$. 
Applying the described above procedure, one obtains in the three-loop approximation [72] $\beta$ - and $\gamma$-functions in the form [73]:

$$
\begin{aligned}
\beta_{u}(u, v)= & -(4-d) u\left\{1-u-\frac{12}{m n+8} v+\frac{8}{(m+8)^{2}} \times\right. \\
& {\left[(5 m+22)\left(i_{1}-\frac{1}{2}\right)+(m+2) i_{2}\right] u^{2}+\frac{96}{(m+8)(m n+8)} \times } \\
& {\left[(m+5)\left(i_{1}-\frac{1}{2}\right)+\frac{m+2}{6} i_{2}\right] u v+\frac{24}{(m n+8)^{2}} \times } \\
& {\left.\left[(m n+14)\left(i_{1}-\frac{1}{2}\right)+\frac{m n+2}{3} i_{2}\right] v^{2}+\beta_{u}^{(3 L A)}+\ldots\right\} } \\
\beta_{v}(u, v)= & -(4-d) v\left\{1-v-\frac{2(m+2)}{m+8} u+\frac{8}{(m n+8)^{2}} \times\right. \\
& {\left[(5 m n+22)\left(i_{1}-\frac{1}{2}\right)+(m n+2) i_{2}\right] v^{2}+\frac{96(m+2)}{(m+8)(m n+8)} \times } \\
& {\left.\left[i_{1}-\frac{1}{2}+\frac{i_{2}}{6}\right] u v+\frac{24(m+2)}{(m+8)^{2}}\left[i_{1}-\frac{1}{2}+\frac{i_{2}}{3}\right] u^{2}+\beta_{v}^{(3 L A)}+\ldots\right\} } \\
& \left.\left.\frac{2(m n+2)}{(m n+8)^{2}} v^{2}\right] i_{2}+\gamma_{\phi}^{(3 L A)}+\ldots\right\} \\
\gamma_{\phi}(u, v)= & 2(4-d)\left\{\left[\frac{2(m+2)}{(m+8)^{2}} u^{2}+\frac{4(m+2)}{(m+8)(m n+8)} u v+\right.\right. \\
& \left.\left.\frac{2(m-d)\left\{\frac{m+2}{m+8} u+\frac{m n+2}{m n+8} v-12\left[\frac{m+2}{(m+8)(m n+8)^{2}} u^{2}+\right.\right.}{(m)} u v+\frac{m n+2}{(m n+8)^{2}} v^{2}\right]\left(i_{1}-\frac{1}{2}\right)+\bar{\gamma}_{\phi^{2}}^{(3 L A)}+\ldots\right\}
\end{aligned}
$$

Here $d$ is space dimension, $m$ is order parameter component number, $n$ is replica index, $i_{1}$ and $i_{2}$ are dimensionally dependent two-loop integrals. The corresponding coefficients for three-loop parts are listed in the Appendix. The values for the three-loop integrals $i_{3} \ldots i_{8}$ which appear in three-loop coefficients for integer $d=2,3$ are listed in [74]. In particular, substituting loop integrals $i_{1}, i_{2}$, as well as $i_{3}, \ldots, i_{8}$ in (2.15)-(2.18) by their values at $d=3$ we get at $n=0, m=1$ the corresponding functions of the $3 d$ weakly diluted Ising model, which in the 3-loop approximation were obtained in [35]. At $d=3, m, n$ - arbitrary corresponding expressions coincide with those, obtained for the $3 d$ anisotropic $m n$-vector model in [75]. Our idea is to keep the dimensional dependence of the loop integrals and, being based on their numerical values for arbitrary $d[24]$, to study the $O(m n)$-model at arbitrary (non-integer) $d$ as well. But for the reason explained above, the point of main interest here will be the replica limit $n=0$ of the anisotropic $m n$-vector model, especially the case $m=1$.

Expressions for $\beta$ - and $\gamma$-functions will be the starting point for the qualitative study of the main features of the critical behaviour which will be done in the next section. 


\section{Resummation}

As we have already mentioned, the values of the $\gamma$-functions in a fixed point $\left(u^{*}, v^{*}\right)$ lead to the values of the critical exponents $\eta$ and $\nu$. However, it is well known now that the series for $R G$-functions are of asymptotic nature [76-78] and imply the corresponding resummation procedure to extract reliable data on their basis. Let us note, however, that, as to our knowledge, the asymptotic nature of the series for $R G$-functions has been proved only for the case of the model with one coupling [79], and the application of a resummation procedure to the case of several coupling constants is based rather on general belief than on a proved fact. Dealing with a series of a zero radius of convergence, one needs to apply a resummation procedure to reconstruct functions in a non-zero region of convergence. The idea of such a resummation method which can be applied to an asymptotic series consists in changing the order of passing to the limit [80]. In the case of one variable it means that, being based on the classic definition of a power series sum

$$
S(x)=\lim _{n \rightarrow \infty} \sum_{i=1}^{n} a_{i} x^{i},
$$

one performs an identical transformation [81]

$$
\begin{aligned}
& S(x) \equiv \lim _{n \rightarrow \infty} \sum_{i=1}^{n}\left(\frac{a_{i} x^{i}}{i !} \int_{0}^{\infty} d t \exp (-t) t^{i}\right) \equiv \\
& \lim _{n \rightarrow \infty}\left\{\lim _{A \rightarrow \infty} \int_{0}^{A} \mathrm{~d} t\left[\sum_{i=1}^{n} \frac{a_{i}(x t)^{i}}{i !} \exp (-t)\right]\right\},
\end{aligned}
$$

and by a new definition of the sum one understands the expression:

$$
\begin{gathered}
S^{\prime}(x)=\lim _{A \rightarrow \infty}\left\{\lim _{n \rightarrow \infty} \int_{0}^{A} \mathrm{~d} t\left[\sum_{i=1}^{n} \frac{a_{i}(x t)^{i}}{i !} \exp (-t)\right]\right\} \equiv \\
\int_{0}^{\infty} \mathrm{d} t \exp (-t) \sum_{i=1}^{\infty} \frac{a_{i}(x t)^{i}}{i !},
\end{gathered}
$$

where $\sum_{i=1}^{n} a_{i}(x t)^{i} / i$ ! is called the Borel image of $\sum_{i=1}^{n} a_{i} x^{i}$. Such a trick is natural in a sense that in the case of a convergent series $S^{\prime}=S$ within its radius of convergence.

The above mentioned procedure in the case of one variable is known as the Borel resummation technique and in different modification is widely used in the studies of asymptotic series. Unfortunately such a technique cannot be applied in our case because only truncated sums of the series are known. To get over this obstacle one represents the Borel image of the initial sum in the form of a rational approximant and in such a way reconstitutes the general term of the series. The technique which involves a rational approximation and the Borel transformation together is known as the Padé-Borel resummation technique (in the field-theoretical $R G$ content see $[82,83]$ as an example of its application).

Note here that the resummation technique, based on the conformal mapping, which is widely used in the theory of critical phenomena [84], cannot be applied in our case because its application postulates information on the high order behaviour of the series for $\beta$ - and $\gamma$-functions. The latter is still unknown for the theory with the Lagrangian (2.1).

We recall that in the case of one coupling the Padé-Borel resummation is performed as follows:

- constructing the Borel-image of the initial sum:

$$
\sum_{i=1}^{n} a_{i} x^{i} \Rightarrow \sum_{i=1}^{n} \frac{a_{i}(x t)^{i}}{i !}
$$


- the Borel-image is extrapolated by a rational approximant

$$
[M / N]=[M / N](t) ;
$$

here by $[M / N]$ one means the quotient of two polynomials in $x t ; M$ is the order of the numerator and $N$ is that of the denominator;

- the resummed function is obtained in the form:

$$
S^{r e s}=\int_{0}^{\infty} \mathrm{d} t \exp (-t)[M / N](t) .
$$

In the two variables case only the first step is changed; namely, here we define the Borel image as

$$
\sum_{i, j} a_{i, j} x^{i} y^{j} \Rightarrow \sum_{i, j} \frac{a_{i, j}(x t)^{i}(y t)^{j}}{(i+j) !} .
$$

Generalization to the many variable case is trivial.

Now one can easily see that not only one way of resummation can be devised. First of all, an arbitrariness appears when noticing the $\beta$-functions to have non-trivial prefactors which depend on the variables $u$ and $v$ and thus may or may not be involved into resummation. Being taken into account, the latter ambiguity leads to the generalization of a resummation procedure - instead of the Borel image we construct the so-called Borel-Leroy image:

$$
\sum_{i=1}^{n} a_{i} x^{i} \Rightarrow \sum_{i=1}^{n} \frac{a_{i}(x t)^{i}}{\Gamma(i+p+1)},
$$

where $\Gamma(x)$ is the Euler's gamma function, and after performing the second step (3.23) in the resummation procedure the new sum is defined via:

$$
S^{r e s}=\int_{0}^{\infty} \mathrm{d} t \exp (-t) t^{p}[M / N](t),
$$

here $p=0$ when taking $\beta$-functions without prefactors, $p=1$ - with prefactors. But nothing prevents us from considering $p$ to be an arbitrary number.

The second arbitrariness rises up from the fact that the Borel-Leroy image of the initial sum may be represented by a rational approximant in different ways. Thus, one can write down its various Padé approximants in the single variable $t$. Within the three-loop approximation these are [2/1], $[1 / 2]$ and $[0 / 3]$ approximants.

On the other hand, it is also possible to take into account the fact that $\beta$ - and $\gamma$-functions of the problem under consideration actually depend on two variables and, therefore, Chisholm approximants may be used. They are the generalization of Padé approximants to the case of two variables [85]. Chisholm approximant of type $[M / N](u, v)$ is defined as a quotient of two polynomials both in $u$ and $v$, of degrees $M$ and $N$, so that the first terms of its expansion are equal to those of the function which is being approximated.

The way of the resummation procedure, when Chisholm approximant of the Borel-Leroy image is constructed, is referred to as the Chisholm-Borel resummation technique. Generally speaking, Chisholm approximants in our 
case can be of type [3/1], [2/2], [1/3] and [0/4], but none of them is unique now. One can easily comprehend the reason on an example of constructing an approximant [3/1]. Being by definition the quotient of two polinomials of degree 3 and 1 , in general it looks like:

$$
\begin{gathered}
{\left[1+a_{10} u+a_{01} v+a_{20} u^{2}+a_{11} u v+a_{02} v^{2}+\right.} \\
\left.a_{30} u^{3}+a_{21} u^{2} v+a_{12} u v^{2}+a_{03} v^{3}\right] /\left[1+b_{10} u+b_{01} v\right]
\end{gathered}
$$

thus having $2+3+4+2=11$ variables $a_{i, j}$ and $b_{i, j}$ to be defined. In its turn the input expression, namely any of the functions (2.15-2.18), has the structure:

$$
1+d_{10} u+d_{01} v+d_{20} u^{2}+d_{11} u v+d_{02} v^{2}+d_{30} u^{3}+d_{21} u^{2} v+d_{12} u v^{2}+d_{03} v^{3}
$$

and thus guarantees only 11-2 $=9$ equations for $a_{i, j}$ and $b_{i, j}$. This means that 2 additional conditions should be imposed. These could be:

$$
a_{3,0}=a_{0,3}=0
$$

or

$$
a_{2,1}=a_{1,2}=0
$$

or any other conditions. There exists no particular reason which can make us prefer any one of them. Except for some general suggestions.

Summarizing what has been said, one can state that all the above described resummation methods, depending on the type of the applied approximant, may be divided into two large groups: the Padé-Borel and the Chisholm-Borel method. Any of them can differ by degrees $M$ and $N$ of polynomials in the numerator and the denominator of the respective rational approximant $[M / N]$. For the Chisholm-Borel method even for given $M$ and $N$ the appropriate approximant is not unique. In addition, an arbitrary parameter $p$ should be noted (see eq.3.26).

One would have to apply different resummation frameworks in order to obtain reliable results and find which of the methods is the most effective. However, strong restriction on the number of choices can be imposed. Further in this Section we are going to discuss this in detail.

First of all, there are some considerations concerning the order of polynomials in the approximant $[M / N]$. The series for $\beta$ - and $\gamma$-functions are sign-alternating, also in the asymptotical regime. This is confirmed by the asymptotics of the series for the $R G$-functions in the case $m=1, n=2$ and $n=3$ which was found in [86]. The approximant generating such a series might be chosen in the form $[M / 1]$ with the positive coefficients at the variable $t$ (or $u$ and $v$ ). Choosing an approximant with a non-linear denominator, generally speaking, one does not ensure the desired properties. Direct calculations affirm the argumentation: $\beta$-functions, resummed with the Padé-Borel and the Chisholm-Borel methods with approximants $[M / N], N>1$, for $u<0, v>0$ give the roots which lie far from the expected values, which for $d=3$ are known up to the order of four loops [39], and for general $d$ were calculated from the two-loop $\beta$-functions [68]. This is true for any $p$. The stated results permit us to eliminate from our consideration approximants with a non-linear denominator.

Note as well that choosing representation of the $R G$-functions (2.15)(2.18) in the form of Padé or Chisholm approximant might result in the 
appearance of a pole in the obtained expression. In the present study we will avoid such a situation. In particular, this leads to the fact that the Chisholm-Borel method of resummation is preffered, as far as it allows us to proceed in a wide range of the space dimension without the appearance of poles. Treating the task in this way and comparing the results obtained within the frames of the Padé-Borel and the Chisholm-Borel methods one notes that there exists an upper bound of the dimension, above which there are no solutions for the resummed $\beta$-functions. The upper bound depends on a resummation method and on an order of loop approximation. When applying the Chisholm-Borel method, the progress from the 2-loop [68] to the 3-loop approximation increases the bound from $d=3.5$ to $d=3.8$; the application of the Padé-Borel method, on the contrary, decreases the bound from $d=3$ to $d=2.1$ in the respective loop level.

So, the results given below are obtained by the Chisholm-Borel method applied to the approximant of type [3/1]. In order to determine the form of the approximant completely one must define two additional conditions, already mentioned. Here there also exist some general suggestions which can make us prefer some kind of additional equations. Firstly, they are expected to be symmetric in variables $u$ and $v$, otherwise, the properties of the symmetry related to these variables would depend, except for the properties of the Lagrangian, on the method of calculation. Secondly, it is obvious that by the substitution $v=0$ all the equations which describe the critical behaviour of the diluted model are converted into appropriate equations of the pure model. However, if a pure model is solved independently, the resummation technique with the application of Padé approximant is used. Thus, Chisholm approximant is to be chosen in such a way that, by putting any of $u$ or $v$ equal to zero, one obtains Padé approximant for a one-variable case. This also implies a special choice of additional conditions. In the present study amidst all the possible expressions which satisfy the stated demand we choose Chisholm approximant $[3 / 1]$ by putting coefficients at $u^{3}$ and $v^{3}$ to be equal to zero (see eq. 3.30).

\section{Results}

Now we are going to apply the mathematical framework which was discussed in previous sections in order to obtain numerical characteristics of the critical behaviour of the weakly-diluted Ising model in general dimensions. We will also confirm quantitatively some general suggestions concerning resummation methods, mentioned so far.

It was noted in Section 1 that the critical behaviour of the quenched weakly-diluted Ising model is described by the effective Lagrangian (2.1) in the case $m=1$ and a zero replica limit. Namely, the task in the end comes to obtaining fixed points which are defined by simultaneous zero of the both $\beta$-functions. Among all the possible fixed points we are interested only in those in the ranges $u^{*}>0, v^{*} \leqslant 0$ and only in stable ones where the stability means that two eigenvalues $b_{1}, b_{2}$ of the stability matrix $B=\partial \beta_{u_{i}} /\left.\partial u_{j}\right|_{u_{i}^{*}}$, $u_{i} \equiv\{u, v\}$ are positive or possess positive real parts. The structure of the $\beta$-functions (2.15)-(2.16) yields the possibility of four solutions for the fixed points. The first two $\left\{u^{*}=0, v^{*}=0\right\}$ and $\left\{u^{*}=0, v^{*}>0\right\}$ in our case at $d<4$ are out of physical interest, while the second pair which consists of pure $\left\{u^{*}>0, v^{*}=0\right\}$ and mixed $\left\{u^{*}>0, v^{*}<0\right\}$ points, are responsible for two possible critical regimes. The critical behaviour of the diluted model coincides with that of the pure model when the pure fixed point appears to be stable. If the mixed point is stable, the new 
(diluted) critical behaviour of the system takes place. The type of the critical behaviour depends on the number $m$ of the order parameters components and on the dimensionality $d$ : at any $d, 2 \leqslant d<4$ a system with large enough $m$ is not sensitive to the weak dilution in the sense that asymptotic values of critical exponents do not change; only starting from some marginal value $m_{c}$, at $m<m_{c}$ the mixed fixed point becomes stable and the crossover to the random critical behaviour occurs. The problem of determining $m_{c}$ as a function of $d$ will be discussed later. Now we would like to state that $m_{c} \geqslant 1$ for any $d, 2 \leqslant d<4$, and, thus, just the mixed fixed point governs the asymptotic critical behaviour of the the diluted Ising model.

If one attempts to find the fixed points from the $\beta$-functions (2.15)(2.16) without resummation, there always appears only the Gaussian $\left\{u^{*}=\right.$ $\left.0, v^{*}=0\right\}$ trivial solution; the existence of the rest possible three fixed points depends on the concrete details of the $\beta$-functions portions in the braces in expressions $(2.15)-(2.16)$. In a $3 d$ case it appears that without a resummation the non-trivial mixed fixed point does not exist in one-, twoand four-loop approximations $[39,40]$. It is only the three-loop approximation where all the four solutions of the set of equations (2.12) exist [35]. In figure 1 we show the behaviour of the non-resummed $\beta$-functions of the three-dimensional weakly diluted Ising model in the three-loop approximation. Resummed functions are shown in the same approximation in figure 2. The shape of the functions remains alike in the region of $\beta_{u}=\beta_{v}=0$. The fixed points correspond to the crossing of the lines $\beta_{u}=0, \beta_{v}=0$ as it is demonstrated in figures 3,4 . The left-hand column in figures 3,4 shows the lines of zeros of non-resummed $\beta$-functions in three dimensions in one-, two-, three- and four-loop (results of $[39,40]$ ) approximations. The thick line corresponds to the roots of the $\beta_{u}$-function, the circles depict zeros of the $\beta_{v}$-function; the thin solid and dashed lines show the roots of analytically continued $\beta_{u^{-}}$and $\beta_{v}$-functions respectively. One can see in the figures that all non-trivial solutions are obtained only within the three-loop level of the perturbation theory. In the next order all fixed points disappear which is a strong evidence of their accidental origin. At any arbitrary $d, 2 \leqslant d<4$ the qualitative behaviour of the functions is very similar to that shown in figures 3 and 4 .

As it has already been mentioned, in order to reestablish the lost pure and mixed points one applies the resummation procedure to $\beta$-functions. In the three-dimensional space the result of resummation is illustrated by the right-hand column in figures 3 and 4 . Here we have used the ChisholmBorel resummation technique choosing Chisholm approximant in the form discussed in the previous Section with $p=1$ in successive approximation in the number of loops. The icons in the figures which correspond to a oneloop level are the visual proof of the degeneracy of $\beta$-functions in this order of the perturbation theory: the graphs of root-lines are parallel independently of resummation. The rest three images in the right-hand columns are a good graphic demonstration of the reliability of the Chisholm-Borel resummational method: two-, three- and four-loop pictures are quantitatively similar, the coordinates of the pure and mixed point are close.

The numerical results of our study are given in table 1 . Here the coordinates of the stable mixed fixed point, as well as of the critical exponents of the quenched weakly diluted Ising model are listed as functions of $d$ between $d=2$ and $d=3.8$. The eigenvalues $b_{1}$ and $b_{2}$ of the stability matrix are given as well.

It was already noted that the values of $\gamma$-functions in a stable point yield the numerical characteristics of the critical behaviour of the model. For 


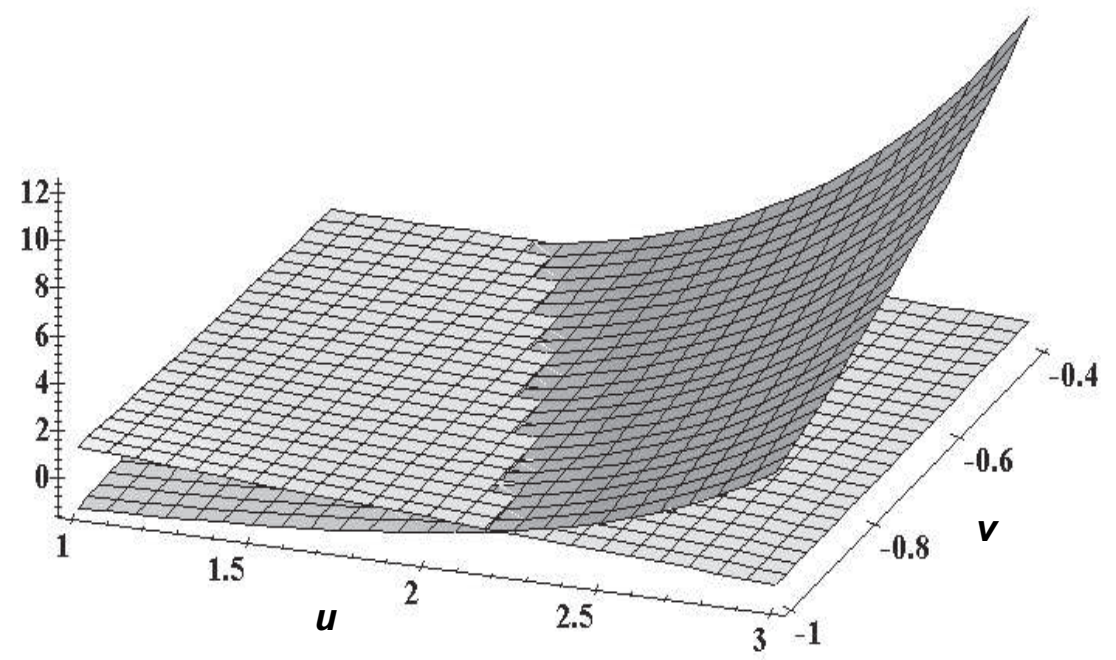

Figure 1. The non-resummed $\beta$-functions in the three-loop approximation; $d=3, m=1, n=0$.

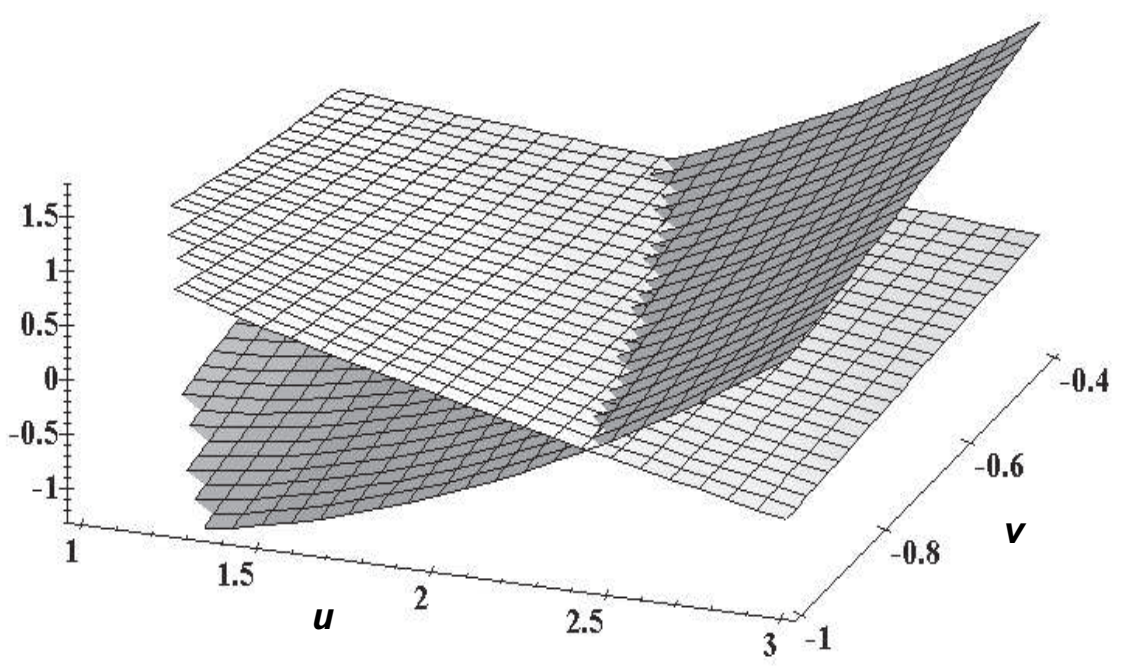

Figure 2. The Chisholm-Borel resummed $\beta$-functions in the three-loop approximation; $d=3, m=1, n=0$. 


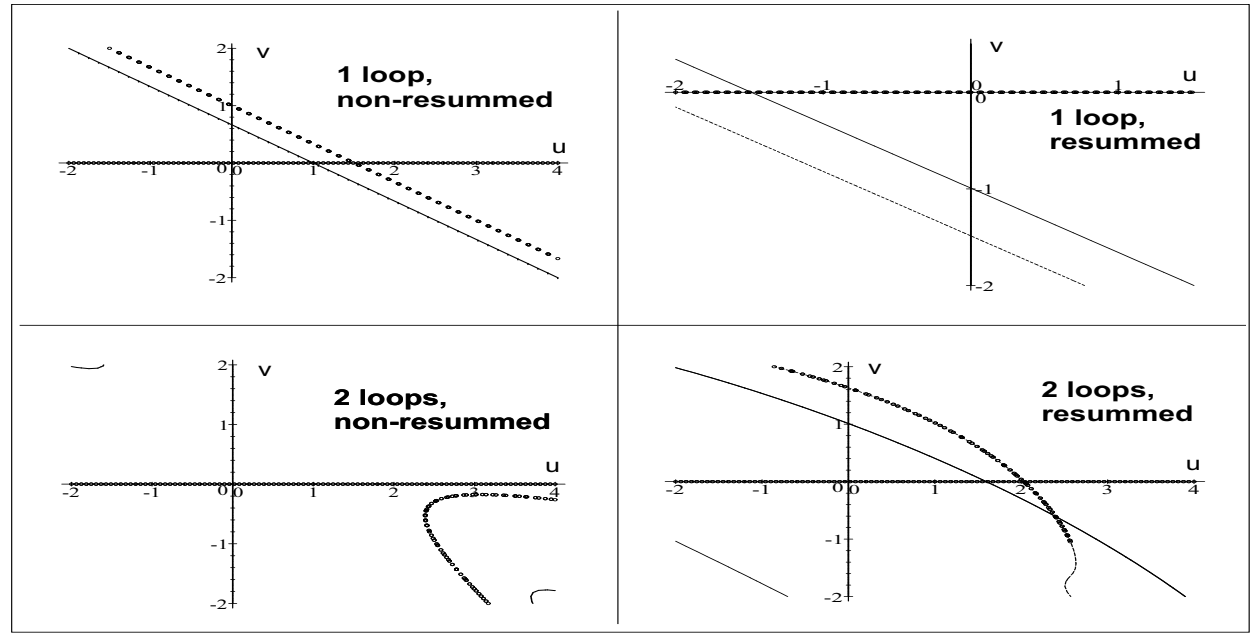

Figure 3. The lines of zeros of non-resummed (left-hand column) and resummed by the Chisholm-Borel method (righthand column) $\beta$-functions for $m=1, n=0$ in different orders of the perturbation theory: one- and two-loop approximations. Circles correspond to $\beta_{u}=0$, thick lines depict $\beta_{v}=0$. Thin solid and dashed lines show the roots of the analytically continued functions $\beta_{u}$ and $\beta_{v}$ respectively. One can see the appearance of the mixed fixed point $u>0, v<0$ in the two-loop approximation for the resummed $\beta$-functions.

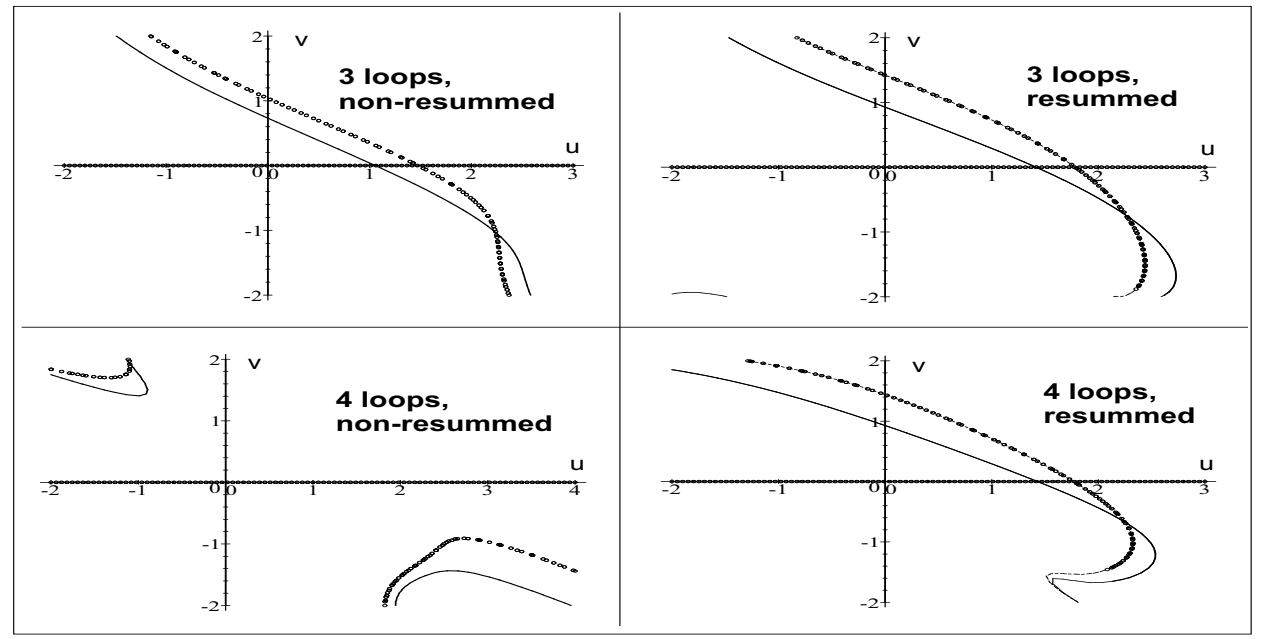

Figure 4. The lines of zeros of non-resummed (left-hand column) and resummed by the Chisholm-Borel method (righthand column) $\beta$-functions for $m=1, n=0$ in threeand four-loop approximations. The notations are the same as in figure 3 . Close to the mixed fixed point the behaviour of the resummed functions remains alike with the increase of the order of approximation. This is not the case for non-resummed functions. 
Table 1. The stable point coordinates, critical exponents and the eigenvalues of the stability matrix of the weakly diluted Ising model at arbitrary $d$. The three-loop approximation (the $c$-subscript denotes that real parts of the corresponding eigenvalues are given).

\begin{tabular}{lllllllll}
\hline \hline$d$ & $u^{*}$ & $v^{*}$ & $\gamma$ & $\nu$ & $\alpha$ & $\eta$ & $b_{1}$ & $b_{2}$ \\
\hline & & & & & & & & \\
2.0 & 2.0268 & -0.2802 & 1.8395 & 0.9664 & 0.0673 & 0.0965 & 0.2176 & 1.5189 \\
2.1 & 2.0327 & -0.3156 & 1.7679 & 0.9229 & 0.0620 & 0.0843 & 0.2373 & 1.4608 \\
2.2 & 2.0412 & -0.3523 & 1.7026 & 0.8838 & 0.0558 & 0.0734 & 0.2562 & 1.4011 \\
2.3 & 2.0525 & -0.3908 & 1.6427 & 0.8484 & 0.0488 & 0.0637 & 0.2742 & 1.3395 \\
2.4 & 2.0671 & -0.4312 & 1.5875 & 0.8162 & 0.0411 & 0.0550 & 0.2913 & 1.2759 \\
2.5 & 2.0854 & -0.4740 & 1.5364 & 0.7868 & 0.0330 & 0.0472 & 0.3074 & 1.2100 \\
2.6 & 2.1081 & -0.5196 & 1.4890 & 0.7598 & 0.0245 & 0.0402 & 0.3226 & 1.1418 \\
2.7 & 2.1359 & -0.5687 & 1.4449 & 0.7349 & 0.0157 & 0.0340 & 0.3370 & 1.0709 \\
2.8 & 2.1698 & -0.6219 & 1.4036 & 0.7119 & 0.0068 & 0.0284 & 0.3505 & 0.9971 \\
2.9 & 2.2113 & -0.6803 & 1.3648 & 0.6905 & -0.0023 & 0.0234 & 0.3635 & 0.9197 \\
3.0 & 2.2621 & -0.7454 & 1.3283 & 0.6705 & -0.0115 & 0.0189 & 0.3764 & 0.8380 \\
3.1 & 2.3250 & -0.8190 & 1.2938 & 0.6518 & -0.0205 & 0.0150 & 0.3905 & 0.7504 \\
3.2 & 2.4039 & -0.9038 & 1.2611 & 0.6342 & -0.0295 & 0.0116 & 0.4095 & 0.6528 \\
3.3 & 2.5044 & -1.0040 & 1.2299 & 0.6176 & -0.0381 & 0.0086 & 0.4653 & 0.5127 \\
3.4 & 2.6359 & -1.1259 & 1.2001 & 0.6019 & -0.0464 & 0.0061 & $0.4436 \mathrm{c}$ & $0.4436 \mathrm{c}$ \\
3.5 & 2.8140 & -1.2804 & 1.1714 & 0.5869 & -0.0541 & 0.0040 & $0.3946 \mathrm{c}$ & $0.3946 \mathrm{c}$ \\
3.6 & 3.0678 & -1.4869 & 1.1436 & 0.5724 & -0.0608 & 0.0023 & $0.3411 \mathrm{c}$ & $0.3411 \mathrm{c}$ \\
3.7 & 3.4570 & -1.7849 & 1.1160 & 0.5583 & -0.0657 & 0.0010 & $0.2822 \mathrm{c}$ & $0.2822 \mathrm{c}$ \\
3.8 & 4.0852 & -2.2303 & 1.0870 & 0.5436 & -0.0656 & 0.0003 & $0.2136 \mathrm{c}$ & $0.2136 \mathrm{c}$ \\
\hline \hline
\end{tabular}

example, given the resummed functions $\gamma_{\phi}^{\text {Res }}$ and $\bar{\gamma}_{\phi^{2}}^{\text {Res }}$, the pair of equations

$$
\begin{aligned}
& \gamma_{\phi}^{\text {Res }}\left(u^{*}, v^{*}\right)=\eta \\
& \bar{\gamma}_{\phi^{2}}^{\text {Res }}\left(u^{*}, v^{*}\right)=2-\nu^{-1}-\eta
\end{aligned}
$$

allows us to find the exponents $\eta$ and $\nu$. All other exponents can be obtained from the familiar scaling laws.

However, one can proceed in a different way. That is, by means of the scaling laws it is possible to reconstitute the expansion in coupling constans of any exponent of interest or of any combination of exponents, and only after that to apply the resummation procedure. If exact calculations were performed, the answer would not depend on the sequence of operations. However, this is not the case for the present approximate calculations. We have chosen the scheme of computing where the resummation procedure was applied to the combination $\nu^{-1}-1=1-\bar{\gamma}_{\phi^{2}}-\gamma_{\phi}$ and $\gamma^{-1}=\left(2-\bar{\gamma}_{\phi^{2}}-\right.$ $\left.\gamma_{\phi}\right) /\left(2-\gamma_{\phi}\right)$. The exponents $\alpha, \beta$ and $\eta$ have been calculated on the basis of numerical values of the exponents $\gamma$ and $\nu$. The resummation scheme is quite insensitive to the choice of the parameter $p$ given by (3.26)-(3.27). We visualize this by figure 5 where the magnetic susceptibility critical exponent $\gamma$ is plotted as a function of $p$ within the three-loop approximation in the three-dimensional space. The value of $\gamma$ increases by about $0.08 \%$ when passing from $p=0$ to $p=10$ which is below the expected accuracy of the exponent. This permits us not to pay special attention to the parameter 
$p$. However, we should remark that all output computations have been performed here, as well as in [68], with $p=1$. The dependence of other critical exponents on $p$ yields the same order of accuracy for them.

One can see in table 1 that the chosen way of calculation yields the results which are in good agreement with the data of recent studies. For example, at $d=2$ the critical behaviour of the weakly diluted quenched Ising model is assumed to coincide with that of the pure model. Our computations confirm the conjecture: the exponent $\gamma$ differs from the exact value $7 / 4$ by the order of $5 \%$, the exponent $\nu$ is smaller from the expected value less than by $4 \%$. It is also interesting to compare these numbers with those obtained within the 2-loop approximation [68]: all the exponents of the three-loop level lie slightly farther from the expected exact values of Onsager than those of the two-loop approximation. This may be explained by the oscilatory nature of the approach to the exact values depending on the order of the perturbation theory. It is also interesting to note that the two-loop approximation yields better estimates for the heat capacity critical exponent $\alpha$ for all $d$ in the range under consideration. Namely, in accordance with the Harris criterion, the $\alpha$-exponent for the diluted Ising system should remain negative. This picture is confirmed much better by the two-loop approximation where $\alpha$ is negative in the whole range of $d$, unlike the threeloop level of the perturbation theory, the results of which yield $\alpha>0$ for $2 \leqslant d \leqslant 2.8$.

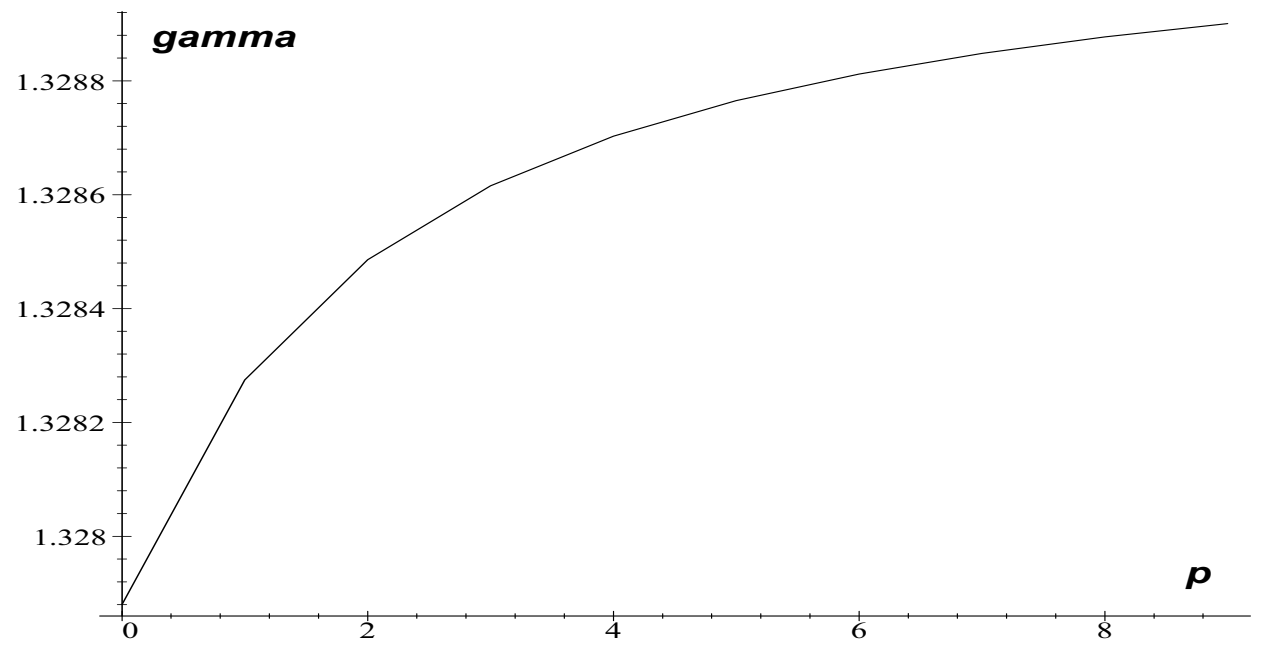

Figure 5. The dependence of the susceptibility exponent $\gamma$ of the three-dimensional weakly diluted Ising model on the choice of the parameter $p$. The calculation has been done by the Chisholm-Borel resummation technique.

However, table 1 shows that the next (third) order does improve our underestanding of the critical behaviour of the model in general dimensions. The results of the two-loop calculations [68] show that starting from some marginal space dimension the approach to the stable point becomes oscilatory: the eigenvalues $b_{1}$ and $b_{2}$ turn to be complex possessing positive real parts. This is an artifact of the calculation scheme and therefore it is expected that by increasing the accuracy of calculations one decreases the region of $d$ which corresponds to the complex eigenvalues. It is realy the case. In the three-loop approximation the region of complex $b_{1}, b_{2}$ is 
bounded from below by $d=3.3$, whereas in the two-loop approximation [68] the corresponding value is lower and is equal to $d=2.9$. Thus, the region of $d$ characterized by the oscilatory approach to the stable fixed point shrinks with the increase of the order of the perturbation theory.

The comparison of the three-dimensional value of $\nu$ with the four-loop result [40] $\nu=0.6701$ gives the accuracy of $0.05 \%$ for our computations (compare with $1 \%$ for two-loops). Thus, it may be stated that the general accuracy of calculations decreases when passing from $d=4$ to $d=2$. In particular, this may be explained by incresing the expansion parameters value $u^{*}$ and $v^{*}$ in the stable point.

The comparison of the present results with the recent studies is provided by figure 6 . Here the behaviour of the correlation length critical exponent $\nu$ obtained by different methods is demonstrated in general dimensions. The results of the massive field-theoretical scheme are plotted by solid (three-loop approximation; the present paper) and dashed (two-loop approximation; ref. [68]) lines. One can see that the two lines practically concide far enough from $d=2$, in particular, both lie very close to the most accurate result for $d=3$ [40] which is shown by the box. However, the three-loop result reconstitutes the latter one with much higher accuracy of

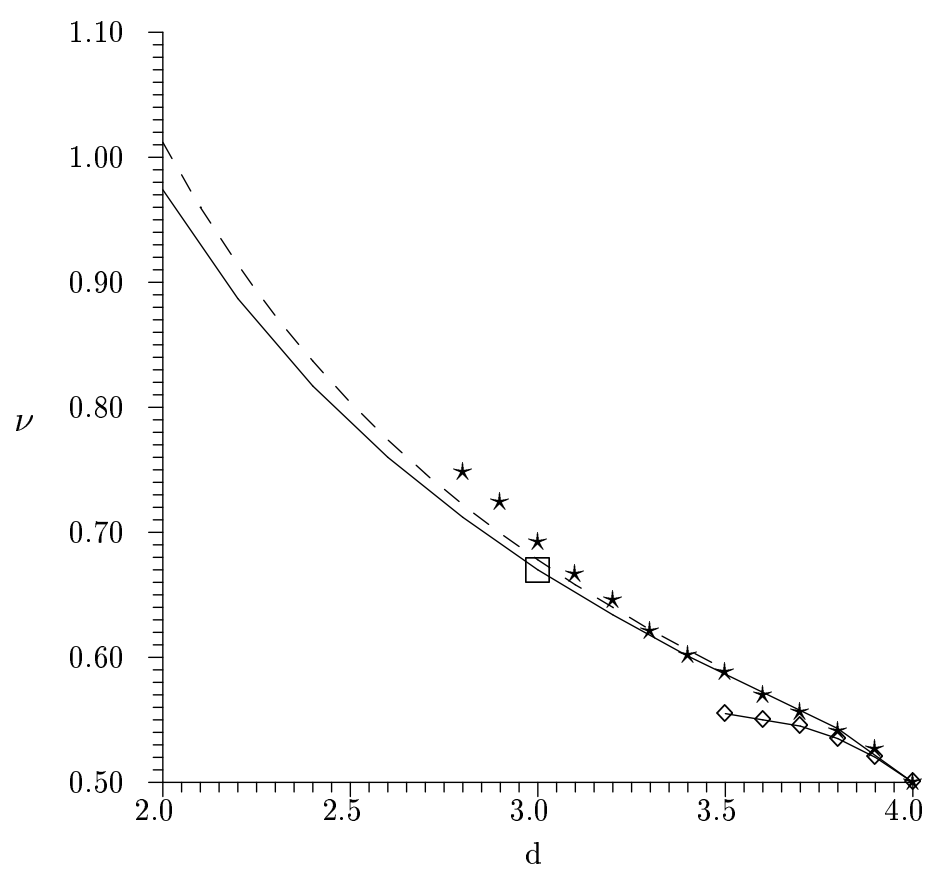

Figure 6. The correlation length critical exponent $\nu$ of the weakly diluted Ising model as a function of the space dimension $d$. The results of two- [68] and three-loop (the present paper) approximations are shown by the dashed and the solid lines respectively, the square reflects the number of the four-loop approximation [40] at $d=3$, asterisks correspond to work [36] and open diamonds refer to the resummed $\sqrt{\varepsilon}$-expansion. 
$0.05 \%$ comparing to $1 \%$ of the two-loop approximation. On the contrary, the exact result of Onsager $\nu=1, d=2$ lies closer to the two-loop approach (see the above consideration).

The application of the scaling-field method [36] yields numbers shown in figure 6 by asterisks. The limit from below $(d=2.8)$ of the method applicability is caused by the truncation of the set of scaling-field equations, which were considered in [36].

One can also attempt to obtain some results by resumming the $\sqrt{\varepsilon \text {-ex- }}$ pansion which is known for the diluted Ising model up to order $O\left(\varepsilon^{3 / 2}\right)$ $[65,66]$. The corresponding results are shown by open diamonds. They were obtained by applying the Padé-Borel resummation scheme to the series of $\sqrt{\varepsilon}$ - expansion $[66,65]$. The value of $\nu$ obtained in such a way is of physical interest only very close to $d=4$. Even in the next orders of the expansions the values of critical exponents are not improved [67]; this is an evidence of the $\sqrt{\varepsilon}$-expansion unreliability in tasks like the one under consideration. To compare, one can state that the situation with the applied in the present paper theoretical scheme is contrary to the $\sqrt{\varepsilon}$-expansion. While the twoloop approximation is valid in ranges $2 \leqslant d<3.4$, the next order of the perturbation theory enlarges the upper bound up to $d=3.8$. One can expect that the next steps within the perturbation theory will permit us to obtain the description of the critical behaviour of the model with enough accuracy for any $d, 2 \leqslant d<4$.

Let us recall now that expressions (2.15)-(2.18) for the $R G$-functions, as well as their three-loop parts listed in the Appendix, allow us to study asymptotic critical properties of the $m n$-vector model with arbitrary $m$ and $n$ in arbitrary $d$ not only for the case $m=1, n=0$. In particular, by keeping $m$ as an arbitrary number and putting $n=0$ one can obtain the numerical estimates for the marginal order parameter component number $m_{c}$ which divides the diluted (governed by the mixed fixed point) asymptotic critical behaviour from the pure one, when the $O(m)$-symmetric fixed point remains stable. In accordance with the Harris criterion, the case $m=m_{c}$ corresponds to zero of the heat capacity critical exponent $\alpha$ of the model. One may extract the value of $m_{c}$ from this condition. However, the above discussed results of the three-loop approximation do not yield enough accuracy for $\alpha$. Alternatively, the mixed fixed point should coincide with the pure fixed point at $m=m_{c}$, which in particular means that $\left.v^{*}\left(m=m_{c}\right)\right|_{\text {mixed }}=0$. The last condition was chosen as a basis of our calculation. The appropriate numbers of the present three-loop approximation (thick solid line) together with the data of the two-loop approximation (dashed line) [68] are shown in figure 7. The result of $\varepsilon$-expansion $m_{c}=4-4 \varepsilon$ is depicted by the thin solid line. In the three-loop approximation we obtain $m_{c}=1.40, d=2$ and $m_{c}=2.12, d=3$. These values are to be compared with the exact results of Onsager which are $m_{c}=1$ at $d=2$, and the theoretical estimate $m_{c}=1.945 \pm 0.002$ [87]. One can see that the two-loop results are closer to the expected values for both $d=2$ and $d=3$. For a two-dimensional case the two-loop value $m_{c}=1.19$ [68] differs from the exact one by $20 \%$, while the three-loop number decreases the accuracy to $40 \%$. The case $d=3, m_{c}>2$ contradicts the suggestion that the $x y$-model asymptotic critical behaviour should not change under dilution in three dimensions. The reason for decreasing the calculation accuracy with increasing the order of the perturbation theory may lie in oscilatory approach to the exact result. Thus, one can expect that already the fourloop case will improve the estimates for $m_{c}$ for all $2 \leqslant d<4$. Let us also note that the determination of $m_{c}$ may serve as a test for improving the 
resummation scheme.

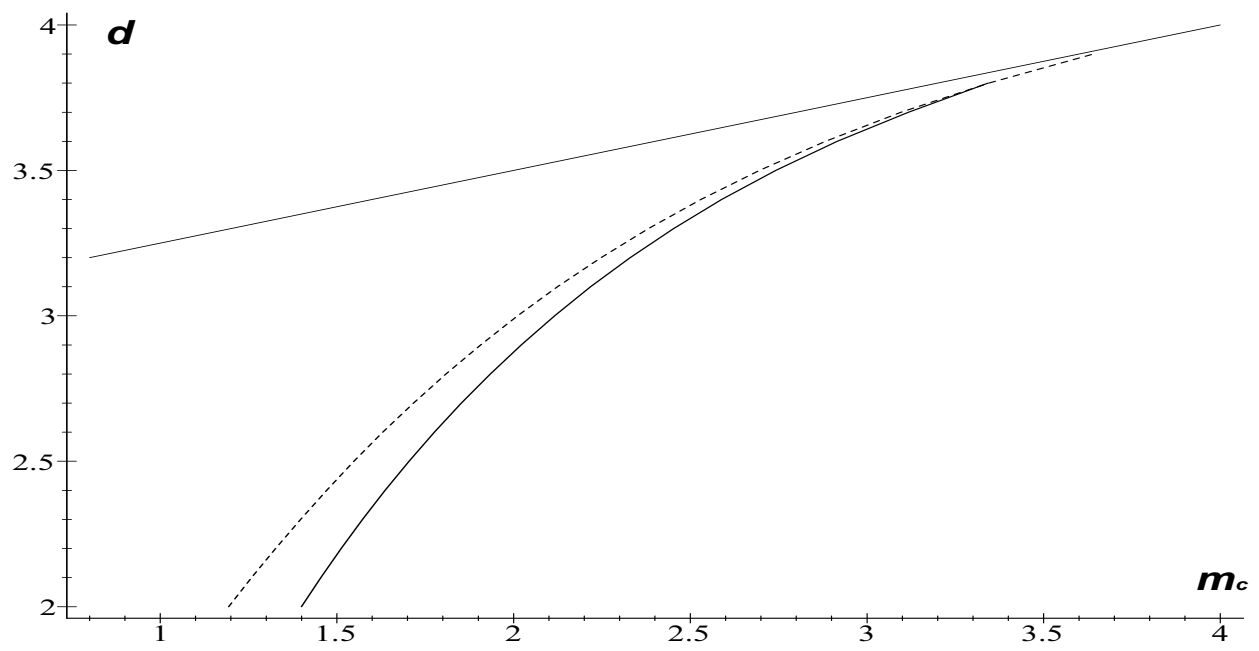

Figure 7. The dependence of the marginal order parameter component number $m_{c}$ on the space dimension $d$. Twoand three-loop results are shown by the dashed and thick solid lines respectively, the $\varepsilon$-expansion data $m_{c}=4-4 \varepsilon$ are depicted by the thin solid line.

\section{Conclusions}

The goal of this paper is to study the critical behaviour of the weakly diluted quenched Ising model in the case when the space dimension $d$ continuously changes from $d=2$ to $d=4$.

As it was mentioned in the Introduction, the study of the pure Ising model at arbitrary $d$, which corresponds to a scalar field-theoretical model with one coupling constant, is the subject of a great deal of papers. It is not the case for the model with a more complicated symmetry. In particular, here we study a model with two couplings corresponding to terms of different symmetry in the Lagrangian (2.1). Up to our knowledge, such a problem was studied previously on the basis of the scaling-field method [36], and field-theoretical fixed dimension renormalization group calculations within a two-loop level of the perturbation theory are available [68].

Our calculations hold within the theoretical scheme of [24,68]. This approach appears to be one amidst other possible calculation schemes for many tasks; however, in our case it seems to have no alternatives within the field-theoretical approach.

Being asymptotic, the resulting series for the $R G$-functions are to be resummed. In the present study we have chosen the Padé-Borel and the Chisholm-Borel resummation techniques. Restricting ourselves to analytic expressions for the resummed functions, we present numerical data mainly obtained on the basis of the Chisholm-Borel resummation technique. Note that the absence of any information on the high-order behaviour of the obtained series for the $R G$-functions does not allow one to apply other resummation schemes, e.g. those based on the conformal mapping technique [84]. 
The quantitative description of the critical behaviour of the model is steady from the point of view of passing from the two- to the three-loop approximation. Smaller agreement between the two- and the three-loop approximations at $d$ far away from $d=4$ may be explained in a way that the precision of computing falls down with the increase of the expansion parameter which takes place at $d \rightarrow 2$. The real parts of eigenvalues corresponding to the mixed point seem to remain positive up to $d=4$, which testifies that at arbitrary $d$ the weakly diluted quenched Ising model is described by the mixed fixed point. Note that performing the resummation scheme as described in this paper, one can make use of the analytic continuation of the resulting expression when a pole in the approximant appears. The results obtained in this way will be presented elsewhere.

\section{Appendix}

Here we have collected the most lengthy expressions for the three-loop contributions to the $R G$-functions. The three-loop part of the $\beta_{u}$-function reads:

$$
\beta_{u}^{3 L A}(u, v)=\beta_{u}^{3,0} u^{3}+\beta_{u}^{2,1} u^{2} v+\beta_{u}^{1,2} u v^{2}+\beta_{u}^{0,3} v^{3},
$$

where

$$
\begin{aligned}
\beta_{u}^{3,0}= & -\frac{1}{(m+8)^{3}}\left[-4\left(31 m^{2}+430 m+1240\right) i_{1}+(m+8)(m+2) \times\right. \\
& \left(-(3 d+8) i_{2}+12\left(i_{3}+i_{8}\right)\right)+48\left(m^{2}+20 m+60\right) i_{4}+ \\
& 24\left(2 m^{2}+21 m+58\right) i_{5}+6\left(3 m^{2}+22 m+56\right) i_{6}+ \\
& \left.24(5 m+22) i_{7}+8\left(4 m^{2}+61 m+178\right)\right] ; \\
\beta_{u}^{2,1}= & -\frac{2}{(m+8)^{2}(m n+8)}\left[-12\left(17 m^{2}+256 m+780\right) i_{1}+\right. \\
& (m+2)\left(-(3 d m+42 d+16 m+80) i_{2}+12(m+14) i_{3}+\right. \\
& \left.18(m+8) i_{8}\right)+24\left(3 m^{2}+70 m+224\right) i_{4}+ \\
& 6\left(15 m^{2}+158 m+448\right) i_{5}+6\left(3 m^{2}+32 m+100\right) i_{6}+ \\
& \left.48(5 m+22) i_{7}+6\left(9 m^{2}+146 m+448\right)\right] ; \\
\beta_{u}^{1,2}= & -\frac{1}{(m+8)(m n+8)^{2}}\left[-12\left(19 m^{2} n+80 m n+470 m+2032\right) i_{1}-\right. \\
& (8(m n+8)(3 d+4)+m(3 d m n+40 m n+78 d+176)) i_{2}+ \\
& 12\left(m^{2} n+8 m n+26 m+64\right) i_{3}+48\left(m^{2} n+8 m n+68 m+\right. \\
& 292) i_{4}+12\left(11 m^{2} n+34 m n+136 m+584\right) i_{5}+ \\
& 6\left(m^{2} n+8 m n+50 m+256\right) i_{6}+576(m+5) i_{7}+ \\
& \left.36(m+2)(m n+8) i_{8}+12\left(5 m^{2} n+22 m n+136 m+584\right)\right] ; \\
\beta_{u}^{0,3}= & -\frac{4}{(m n+8)^{3}}\left[6(m n+10)\left(-2(m n+23) i_{1}+3 i_{6}\right)+\right. \\
& (m n+2)\left(-(4 m n+9 d+8) i_{2}+36 i_{3}+3(m n+8) i_{8}\right)+ \\
&
\end{aligned}
$$




$$
\begin{aligned}
& 72(3 m n+22) i_{4}+9\left(m^{2} n^{2}+14 m n+88\right) i_{5}+ \\
& \left.24(m n+14) i_{7}+3\left(m^{2} n^{2}+38 m n+264\right)\right] .
\end{aligned}
$$

The three-loop part of the $\beta_{v}$-function reads:

$$
\beta_{v}^{3 L A}(u, v)=\beta_{v}^{0,3} v^{3}+\beta_{v}^{1,2} u v^{2}+\beta_{v}^{2,1} u^{2} v+\beta_{v}^{3,0} u^{3},
$$

where

$$
\begin{aligned}
\beta_{v}^{0,3}=- & \frac{1}{(m n+8)^{3}}\left[-4\left(31 m^{2} n^{2}+430 m n+1240\right) i_{1}+(m n+8) \times\right. \\
& (m n+2)\left(-(3 d+8) i_{2}+12\left(i_{3}+i_{8}\right)\right)+48\left(m^{2} n^{2}+\right. \\
& 20 m n+60) i_{4}+24\left(2 m^{2} n^{2}+21 m n+58\right) i_{5}+6\left(3 m^{2} n^{2}+\right. \\
& \left.22 m n+56) i_{6}+24(5 m n+22) i_{7}+8\left(4 m^{2} n^{2}+61 m n+178\right)\right] ; \\
\beta_{v}^{1,2}=- & \frac{4(m+2)}{(m n+8)^{2}(m+8)}\left[-4(28 m n+275) i_{1}-\right. \\
& (3 d m n+4 m n+15 d+56) i_{2}+12(m n+5) i_{3}+ \\
& 24(2 m n+27) i_{4}+3(13 m n+100) i_{5}+6(3 m n+13) i_{6}+ \\
& \left.96 i_{7}+9(m n+8) i_{8}+(29 m n+316)\right] ; \\
\beta_{v}^{2,1}=- & (m n+8)(m+8)^{2}\left[-12(m n+42 m+224) i_{1}-\right. \\
& (3 d m n+12 d m-8 m n+48 d+16 m+256) i_{2}+ \\
& 12(m n+4 m+16) i_{3}+48(5 m+34) i_{4}+ \\
& 12(13 m+56) i_{5}+6(3 m n+14 m+40) i_{6}+ \\
& \left.144 i_{7}+36(m+8) i_{8}+12(11 m+64)\right] ; \\
\beta_{v}^{3,0}= & -\frac{2(m+2)}{(m+8)^{3}}\left[-4(11 m+70) i_{1}-3(d m+2 d+16) i_{2}+\right. \\
& \left.6(m+2)\left(2 i_{3}+3 i_{6}\right)+2(m+8)\left(12 i_{4}+3 i_{5}+3 i_{8}+5\right)\right] .
\end{aligned}
$$

The three-loop part of the $\gamma_{\phi}$-function reads:

$$
\begin{aligned}
\gamma_{\phi}^{3 L A}(u, v)= & -\left[\frac{m+2}{(m+8)^{2}} u^{3}+\frac{3(m+2)}{(m+8)(m n+8)} u^{2} v+\right. \\
& \left.\frac{3(m+2)}{(m+8)(m n+8)} u v^{2}+\frac{m n+2}{(m n+8)^{2}} v^{3}\right]\left(3 i_{8}-4 i_{2}\right) .
\end{aligned}
$$

The three-loop part of the $\bar{\gamma}_{\phi^{2}}$-function reads:

$$
\bar{\gamma}_{\phi^{2}}(u, v)=\bar{\gamma}_{\phi^{2}}^{3,0} u^{3}+\bar{\gamma}_{\phi^{2}}^{2,1} u^{2} v+\bar{\gamma}_{\phi^{2}}^{1,2} u v^{2}+\bar{\gamma}_{\phi^{2}}^{0,3} v^{3},
$$

where

$$
\begin{aligned}
\bar{\gamma}_{\phi^{2}}^{3,0}= & \frac{m+2}{(m+8)^{3}}\left[-4(11 m+70) i_{1}+(m+2)\left(-(3 d-8) i_{2}+\right.\right. \\
& \left.\left.12 i_{3}+18 i_{6}\right)+2(m+8)\left(12 i_{4}+3 i_{5}+5\right)\right]
\end{aligned}
$$




$$
\begin{aligned}
\bar{\gamma}_{\phi^{2}}^{2,1}= & \frac{m+2}{(m+8)^{2}(m n+8)}\left[-12(m n+10 m+70) i_{1}+\right. \\
& (m n+2 m+6)\left(-(3 d-8) i_{2}+12 i_{3}+18 i_{6}\right)+ \\
& \left.6(m+8)\left(12 i_{4}+3 i_{5}+5\right)\right] ; \\
\bar{\gamma}_{\phi^{2}}^{1,2}= & \frac{3(m+2)}{(m+8)(m n+8)^{2}}\left[-4(11 m n+70) i_{1}+\right. \\
& (m n+2)\left(-(3 d-8) i_{2}+12 i_{3}+18 i_{6}\right)+ \\
& \left.2(m n+8)\left(12 i_{4}+3 i_{5}+5\right)\right] ; \\
\bar{\gamma}_{\phi^{2}}^{0,3}= & \frac{m n+2}{(m n+8)^{3}}\left[-4(11 m n+70) i_{1}+\right. \\
& (m n+2)\left(-(3 d-8) i_{2}+12 i_{3}+18 i_{6}\right)+ \\
& \left.2(m n+8)\left(12 i_{4}+3 i_{5}+5\right)\right] .
\end{aligned}
$$

\section{References}

[1] Mandelbrot B. B, Fractals: Form, Chance and Dimension. Freeman, San Francisco, Cal., 1977.

[2] Wilson K. G., Fisher M. E., Phys. Rev. Lett., 1972, vol. 28, No 4, p. 240-243.

[3] Gefen Y., Mandelbrot B. B., Aharony A. // Phys. Rev. Lett., 1980, vol. 45, No 11, p. 855-858.

[4] Gefen Y., Aharony A., Mandelbrot B. B., Kirkpatrick S. // Phys. Rev. Lett., 1981, vol. 47, No 25, p. 1771-1774.

[5] Bonnier B., Leroyer Y., Meyers C. // Phys. Rev. B, 1988, vol. 37, No 10, p. 5205-5210.

[6] Jeżewski W., Tomczak P. // Physica A, 1991, vol. 171, p. 209-222.

[7] Jeżewski W., Tomczak P. // Phys. Lett. A, 1991, vol. 157, No 8-9, p. 507-512.

[8] Jeżewski W. // Physica A, 1994, vol. 210, p. 73-86.

[9] Hu B. // Phys. Rev. B., 1986, vol. 33, No 9, p. 6503-6504.

[10] Wu Y., Hu B. // Phys. Rev. A., 1987, vol. 35, No 3, p. 1404-1411.

[11] Tomczak P. ,Jeżewski W. // Physica A, 1994, vol. 209, p. 275-287.

[12] Le Guillou J. G., Zinn-Justin J. // J. Phys. (Paris), 1987, vol. 48, No 1, p. 19-24.

[13] Katz S. L., Droz M., Gunton J. D. // Phys. Rev. B., 1977, vol. 15, No 3, p. 1597-1599.

[14] Bonnier B., Hontebeurie M. // J. Phys. I (Paris), 1991, vol. 1, p. 331-338.

[15] Novotny M. A. // Europhys. Lett, 1992, vol. 17, No 4, p. 297-302.

[16] Novotny M. A. // Phys. Rev. B, 1992, vol. 46, No 5, p. 2939-2950.

[17] Filippov A. E., Radievskii A. V. // Zh. Eksp. Teor. Fiz., 1992, vol. 102, No 6, p. $1899-1920$.

[18] Filippov A. E. // Teor. Mat. Fiz, 1992, vol. 91, No 2, p. 320-333.

[19] Filippov A. E., Radievskii A.V. // Pism. v Zh. Eksp. Teor. Fiz, 1992, vol. 56, No 2, p. 87-92.

[20] Breus S. A., Filippov A. E. // Physica A, 1993, vol. 192, No 1, p. 486-515.

[21] Parisi G., 1973, in Proceedings of the Cargrése Summer School, unpublished

[22] Parisi G. // J. Stat. Phys., 1980, vol. 23, No 1, p. 49-82.

[23] Holovatch Yu. // Theor. Math. Phys., 1993, vol. 96, No 3, p. 482-495.

[24] Holovatch Yu., Krokhmal's'kii T. // J. Math. Phys., 1994, vol. 35, No 8, p. 3866-3880. 
[25] Fisher M. E., Gaunt D.S. // Phys. Rev., 1964, vol. 133, No 1A, p. A224-A239.

[26] Here so called Fisher renormalization [27] holds. It states that the critical exponents of an annealed system are determined by those of the corresponding pure one.

[27] Fisher M. E. // Phys. Rev., 1968, vol. 176, No 1, p. 257-272.

[28] Harris A. B. // J. Phys. C, 1974, vol. 7, p. 1671-1692.

[29] Birgeneau R. J., Cowley R. A., Shirane G., Joshizawa H. // Phys. Rev. B, 1983, vol. 27, No 11, p. 6747-6753.

[30] Mitchell P. W., Cowley R. A., Yoshizawa H., Böni P., Uemura Y. J., Birgeneau R. J. // Phys. Rev. B, 1986, vol. 34, No 7, p. 4719-4725.

[31] Thurston T. R., Peters C. J., Birgeneau R. J. // Phys. Rev. B, 1988, vol. 37, No 16, p. 9559-9563.

[32] Lubensky T. C. // Phys. Rev. B, 1975, vol. 11, No 9, p. 3573-3580.

[33] Khmelnitskii D. E. // Zh. Eksp. Teor. Fiz., 1975, vol. 68, No 5, p. 1960-1968.

[34] Grinstein G., Luther A. // Phys. Rev. B, 1976, vol. 13, No 3, p. 1329-1343.

[35] Sokolov A. I., Shalaev, B. N. // Fiz. Tv. Tiel., 1981, vol. 23, No 7, p. 20582063.

[36] Newman K. E., Riedel E. K. // Phys. Rev. B, 1982, vol. 25, No 1, p. 264-280.

[37] Jug G. // Phys. Rev. B, 1983, vol. 27, p. 609-612.

[38] Mayer I. O., Sokolov A. I. // Fiz. Tv. Tiel., 1984, vol. 26, No 11, p. 3454-3456.

[39] Mayer I. O., Sokolov A. I., Shalaev B.N. // Ferroelectrics, 1989, vol. 95, p. 93.

[40] Mayer I. O. // J. Phys. A, 1989, vol. 22, p. 2815-2823.

[41] Janssen H. K., Oerding K., Sengespeick E. // J. Phys. A, 1995, vol. 28, No 21, p. $6073-6085$.

[42] Chowdhury D., Staufer D. // J. Stat. Phys., 1986, vol. 44, No 1/2, p. 203-210.

[43] Marro J., Labarta A., Tejada J. // Phys. Rev. B, 1986, vol. 34, No 1, p. $347-349$.

[44] Wang J.-S., Wöhlert M., Mühlenbein H., Chowdhury D. // Physica A, 1990, vol. 166, p. 173-179.

[45] Heuer H.-O. // Phys. Rev. B, 1990, vol. 42, No 10, p. 6476-6484;

Europhys. Lett., 1990, vol. 12, No 6, p. 551-556.

[46] Heuer H.-O. // J. Phys. A, 1993, vol. 26, p. L333-L339.

[47] Holey T., Fähnle M. // Phys. Rev. B, 1990, vol. 41, p. 11709-11712.

[48] Dotsenko Vik. S., Dotsenko Vl. S. // J. Phys. C, 1982, vol. 15, p. 495-507.

[49] Shankar R. // Phys. Rev. Lett., 1987, vol. 58, No 23, p. 2466-2469.

[50] Shalaev B. N. // Fiz. Tv. Tiel., 1988, vol. 30, p. 895.

[51] Ludwig A. W. // Phys. Rev. Lett., 1988, vol. 61, p. 2388.

[52] Shalaev B. N. // Fiz. Tv. Tiel., 1989, vol. 31, p. 93.

[53] Ludwig A. W. W. // Nucl. Phys. B, 1990, vol. 330, p. 639-680.

[54] Shalaev B. N. // Phys. Reports, 1994, vol. 237, No 3, p. 129-188.

[55] Wang J.-S., Selke W., Dotsenko Vl. S., Andreichenko V. B. // Europhys. Lett., 1990, vol. 11, p. 301-305; Physica A, 1990, vol. 164, p. 221-239.

[56] Andreichenko V. B., Dotsenko Vl. S., Selke W., Wang J.-S. // Nuclear. Phys. B, 1990, vol. 344, p. 531-556.

[57] Heuer H.-O. // Phys. Rev B, 1992, vol. 45, No 10, p. 5691-5694.

[58] Talapov A. L., Shchur L. N. Preprints Hep-lat-9404002 and Hep-lat-9404011

[59] Selke W., Shchur L. N., Talapov A. L. Monte Carlo Simulations of Dilute Ising Models, in: Annual Reviews of Computational Physics, ed. D.Stauffer. World Scientific, Singapore (preprint)

[60] Cowley R. A., Birgeneau R. J., Shirane G., Guggenstein H. J., Ikada H. // Phys. Rev. B, 1980, vol. 21, No 9, p. 4038-4048.

[61] Hagen M., Cowley R. A., Nicklow R. M., Ikeda H. // Phys. Rev. B, 1987, vol. 36, No 1, p. 401-408.

[62] Dotsenko Vik. S., Dotsenko Vl. S. // Adv. Phys., 1983, vol. 32, No 2, p. 129-172.

[63] Kim J.-K., Patrascioiu A. // Phys. Rev. Lett., 1994, vol. 72, No 17, p. 27852788.

[64] Golner G., Riedel E. // Phys. Rev. Lett., 1975, vol. 34, p. 856; 
Phys. Lett. A, 1976, vol. 58, p. 11.

[65] Shalaev B. // Zh. Eksp. Teor. Fiz., 1977, vol. 73, p. 3201.

[66] Jayaprakash C., Katz H. J. // Phys. Rev. B, 1977, vol. 16, No 9, p. 3987-3990.

[67] Holovatch Yu., Yavors'kii T., unpublished

[68] Holovatch Yu., Shpot M. // J. Stat. Phys., 1992, vol. 66, No 3/4, p. 867-883.

[69] Brézin E., Le Guillou J. C., Zinn-Justin J. Field theoretical approach to critical phenomena, in Phase transitions and critical phenomena, Vol. 6. Academic Press, New York, 1976

[70] D.J.Amit. Field Theory, the Renormalization Group, and Critical Phenomena. Singapore, World Scientific, 1984.

[71] The subscripts $u, v$ at $\Gamma_{R, u}^{(0,4)}, \Gamma_{R, v}^{(0,4)}$ denote the $O(m n)$-symmetric and hypercubic parts of the four-point vertex function.

[72] Holovatch Yu., unpublished

[73] We have used the change of the notations $(m+8) D u / 6 \rightarrow u,(m n+8) D v / 6 \rightarrow$ $v$ and $6 \beta_{u} /[(m+8) D] \rightarrow \beta_{u}, 6 \beta_{v} /[(m n+8) D] \rightarrow \beta_{v}$ with $\mathrm{D}$ being one loop integral $D=\int \mathrm{d}^{d} q\left(q^{2}+m_{0}^{2}\right)^{-1}$ in order to define convenient numerical scale in which the first two coefficients of the functions $\beta_{u}$ and $\beta_{v}$ are -1 and +1 .

[74] Nickel B. G., Meiron D. I., Baker G. A. Compilation of 2pt and 4pt graphs for continuous spin model. University of Guelph Report, 1977.

[75] Shpot N. A. // Phys. Lett. A, 1989, vol. 142, No 8,9, p. 474-478.

[76] Lipatov L. // Sov. Phys. JETP., 1977, vol. 45, No 2, p. 216-223.

[77] Breźin E., Le Guillou J., Zinn-Justin J. // Phys. Rev. D, 1977, vol. 15, No 6, p. 1544-1557.

[78] Breézin E., Parisi G. // J. Stat. Phys., 1978, vol. 19, No 3, p. 269-292.

[79] Eckmann J.-P., Magnen J., Seneor R. // Commun. Math. Phys., 1975, vol. 39, No 4, p. 251-271.

[80] Hardy G. H. Divergent Series. Oxford, 1948.

[81] Note that for finite number of terms changing of order of integration and summation can always be performed

[82] Baker G. A., Nickel B. G., Green M. S., Meiron D. I. // Phys. Rev. Lett., 1976, vol. 36, p. 1351.

[83] Baker G. A., Nickel B. G., Meiron D. I. // Phys. Rev. B, 1978, vol. 17, No 3, p. $1365-1374$.

[84] Le Guillou J. C, Zinn-Justin J. // Phys. Rev. B, 1980, vol. 21, No 9, p. 3976-3988.

[85] Chisholm J. S. R. // Math. Comp., 1973, vol. 27, p. 841-848.

[86] Kleinert H., Thoms S. // Phys. Rev. D, 1995, vol. 52, No 10, p. 5926-5943.

[87] Bervillier C. // Phys. Rev. B, 1986, vol. 34, No 11, p. 8141-8143.

\title{
ТЕОРЕТИКО-ПОЛЬОВИЙ ПІДХІД ДО ДОСЛІДЖЕННЯ КРИТИЧНОЇ ПОВЕДІНКИ СЛАБО РОЗВЕДЕНОЇ ЗАМОРОЖКЕНОЇ МОДЕЛІ ІЗІНГА ПРИ ДОВІЛЬНІЙ ВИМIРНОСТI ПРОСТОРУ. ТРИПЕТЛЕВЕ НАБЛИЖКЕННЯ
}

\author{
Ю.Головач, Т.Яворський
}

\begin{abstract}
У рамках теоретико польового ренормгрупового підходу при фіксованій вимірності простору у трипетлевому наближенні отримані вирази для $\beta$ - і $\gamma$-функцій анізотропної $m n$-векторної моделі. Розраховані критичні показники слабо розведеної "замороженої" моделі Ізінга та подані оцінки маргінальної кількості компонент параметра порядку $m_{c}$ як функції $d$ у інтервалі $2 \leqslant d<4$. Наведені висновки щодо ефективності різних процедур пересумовування. Результати порівнюються із даними попередніх досліджень.
\end{abstract}

